\title{
Thermochemical and mechanical properties of tea tree (Melaleuca alternifolia) fibre reinforced tapioca starch composites
}

\begin{abstract}
We aim to utilise the tea tree (Melaleuca alternifolia) fibre, a waste from the distillation process, as a reinforcement or filler in tapioca starch (TS) composites. Fabrication of tea tree fibre-reinforced TS composites was successfully developed using a casting method. The physical, thermo-chemical, and mechanical properties were tested in order to get the characterisation of the composites. From the mechanical test, the addition of $5 \%(\mathrm{v} / \mathrm{v})$ tea tree fibre as a filler improved the tensile strength of the TS composites up to $34.39 \%$ in tea tree leaf-reinforced TS composites (TTL/TS), $82.80 \%$ in tea tree branch-reinforced TS composites (TTB/TS) and $203.18 \%$ in tea tree trunk-reinforced TS composites (TTT/TS). The water absorption and swelling of all tea tree fibre-reinforced composites decreased compared to those of TS composites. Most importantly, all parts of the tea tree waste, namely, the tea tree leaf, tea tree branch and tea tree trunk, have additional potential value as fibres that can act as a reinforcement in developing a green biocomposite.
\end{abstract}

Keyword: Biocomposite; Filler; Melaleuca alternifolia; Natural fibre; Reinforcement 